\title{
Studies on the Effect of Diet and Drug Therapies on Serum Ferritin, a Predictor of Type 2 Diabetes Mellitus
}

\author{
P. O. Okonkwo ${ }^{1}$ and Z. S. C. Okoye ${ }^{2}$ \\ ${ }^{1}$,Chemical Pathology Department, Federal College of Veterinary and Medical Laboratory Technology, \\ National Veterinary Research Institute, Vom, Plateau state, Nigeria \\ ${ }^{2}$,Department of Biochemistry, College of Medical Sciences, University of Jos, Jos, Plateau state, Nigeria.
}

\begin{abstract}
Background and Objective: Epidemiological studies have established a link between serum ferritin concentration and type 2 diabetes. This work was carried out to ascertain the effect of therapeutic regimen on the earlier established links.
\end{abstract}

Materials and Methods: Two hundred subjects comprising 130 type 2 diabetics and 70 apparently healthy controls were involved in the survey. Anthropometric parameters, fasting plasma glucose (FPG), Serum levels of ferritin, lipids and biochemical indicators of complications of type 2 DM were determined. Results were analysed statistically by ANOVA, and Pearson correlation.

Results: Serum ferritin, showed a positive correlation with FPG, BP, TC, triglyceride, and TC/HDL ratio among diabetic subjects. With subjects classified based on the antidiabetic therapy, the mean serum ferritin level of the subjects on a combination therapy of drug and diet was significantly $(p<0.05)$ lower than the level in those on either drug or diet alone. Serum ferritin level showed a positive correlation with TC and TC/HDL ratio, in diabetics on drug alone and with PFG, TC, and TG levels in subjects on diet alone but with none in subjects on a combined therapy.

Conclusion: This finding suggests the need to take into due consideration type of diabetic medication when interpreting relationship between iron stores and diabetic risk.

KEY WORDS: diabetes risk factors, diet, combination therapy, ferritin, type 2 diabetes

\section{INTRODUCTION}

Diabetes mellitus is a serious health problem with high rates of incidence and mortality all over the world (1). Globally as of 2010 it was estimated that there were 285 million people with diabetes, type 2 making up about $90-95 \%$ of all diagnosed cases (2). The global pandemic of type 2 diabetes mellitus (T2DM) places an incalculable burden on health care systems (3). Amelioration of T2DM risk usually targets lifestyle and diet, primarily with the aim of reducing obesity, the foremost risk factor in the development of insulin resistance and ultimately T2DM. (3). Established management of T2DM starts with lifestyle changes, i.e, introducing a healthier diet and increasing physical activity in order to improve glucose utilization and promote weight loss. This is accompanied by rapid or even concomitant introduction of an oral antidiabetic agent (4, 5).Epidemiological studies have reported a strong association between elevated serum ferritin concentrations and increased risk for diabetes $(6,7)$. Dongiovianni et al. (8) investigated the effect of iron depletion by deferoxamine on insulin signaling and glucose uptake in HepG2 hepatocytes and in rat liver; deferoxamine was found to induce the constitutive glucose transporter Glut1 and the insulin receptor. Thus, iron depletion by deferoxamine up-regulates glucose uptake and increases insulin receptor activity and signaling in hepatocytesin vitro and in vivo.

However, the generality of the epidemiological studies that have reported strong association between serum ferritin and increased risk for type 2 diabetes appear not to have taken the aetiological effect of the therapeutic regimen into serious consideration when interpreting the observed relationship. Hence, the need to ascertain what effect therapeutic regimen has on the relationship between serum ferritin and type 2 diabetes risks, which is the goal of this study. 


\section{Study location/ Sampling Procedure}

\section{MATERIALS AND METHODS}

This study was carried out amongst diabetic patients attending the outpatient medical clinics of Jos University Teaching Hospital, Plateau Specialist Hospital and ECWA Evangel Hospital Jos. A total of one hundred and thirty (130) type 2 DM patients (70 females and 60 males) aged 40-70 years were involved in the study.

Approval for this study was obtained from the Ethics Committees of JUTH, Plateau Specialist Hospital and ECWA Evangel Hospital Jos. The designing of questionnaire and recruitment of participants was carried out under the close supervision of a clinician, who also allowed access to medical records of diabetic patients from where information as duration of diabetes, type of drug etc was extracted. Informed consent was used in the recruitment of the participants and confidentiality was maintained in accordance with standard medical practice.

\section{Physical Anthropometric Risk Indices}

At the study site, height, weight, and blood pressure of each subject were meseaured by the health workers. Blood pressure was measured by mercury sphygmomanometer in the right upper arm of the subject, who was seated for 5 minutes before the measurement. Blood pressure was measured twice, and the mean of the two measurements was used in the analysis. Weight was measured without shoes to the nearest $0.1 \mathrm{~kg}$ by a weighing machine and height was measured to the nearest $0.1 \mathrm{~m}$ by an anthropometric rod. Body mass index was calculated as weight in kilograms divided by the square of height in metres.

\section{Blood Sample Collection and Preparation}

A $10 \mathrm{ml}$ sample of venous blood was collected from each volunteer after an overnight fast. $2 \mathrm{ml}$ of this aliquot was transferred into a fluoride oxalate bottle for glucose analysis and the remaining into plain tubes for other biochemical analysis. The samples were transported to the laboratory immediately after collection for preparation for the analysis. The sample for blood glucose determination was centrifuged at $3000 \mathrm{rpm}$ for 5 minutes on a digital centrifuge (Biofuge 200; Bosch medical Systems, U.S.A) immediately upon return to the laboratory. The supernatant (Plasma) was used immediately for glucose analysis The portion of blood sample (in plain tube) were allowed to clot at room temperature and then centrifuged at 3000rpm for 5 minutes on a digital centrifuge (Biofuge 200; Bosch Medical Systems, U.S.A) to obtain the serum (supernatant). The serum samples were stored frozen at $-20^{\circ} \mathrm{c}$ pending assay.

\section{Biochemical Analysis}

The parameters that were assayed for include, fasting plasma glucose, serum ferritin, serum total cholesterol, serum triglyceride, serum high density lipoprotein cholesterol (HDL-cholesterol). All parameters were analysed in triplicates. Plasma glucose concentration was determined using the glucose oxidase method described by Trinder (9), Serum Ferritin was assayed using human ferritin enzyme immunoassay test kit (10), serum Serum total cholesterol (TC) and triglyceride (TG) were determined colorimetrically by enzymatic point method of Trinder, (9) while the Serum high-density lipoprotein-cholesterol (HDL-c) was assayed colorimetrically by enzymatic endpoint method (11).

\section{Statistical Analysis}

Statistical analysis was performed with SPSS software version 16.0. Data are expressed as the mean \pm SEM. Results were analysed statistically by student t-test, ANOVA and Pearson correlation coefficient as appropriate. Statistical significance was considered when $\mathrm{p}$ value was less than 0.05 .

\section{RESULTS}

\section{Serum Ferritin Levels in Diabetic Subjects Receiving Different Antidiabetic Therapy}

The results of serum ferritin levels in diabetic subjects receiving different antidiabetic therapy are summarized on Table 1. The mean serum ferritin of diabetic subjects on a combination therapy of oral hypoglycaemic drug and diet $(54.37 \pm 6.21 \mathrm{ng} / \mathrm{ml})$ is significantly lower $(\mathrm{p}<0.05)$ than that of those on either drug $(80.72 \pm 7.12 \mathrm{ng} / \mathrm{ml})$ or diet $(78.52 \pm 9.68)$ therapy alone. This would suggest that a combination (oral drug + diet) therapy is more efficacious in reducing serum ferritin, a predictor of DM, than either oral antidiabetic drug or diet alone- a reduction of $32.64 \%$ and, respectively $30.76 \%$, respectively, compared to those on drug or diet alone. The difference between mean serum ferritin concentration of subjects on oral hypoglycaemic drug alone and that of subjects on diet alone is not statistically significant ( $p>0.05)$. 


\section{Relationship between Serum Ferritin Levels and key Individual Risk Factors in Subjects Receiving Different Therapy}

The results of the analyses of the relationship between serum ferritin levels and blood glucose, physical anthropometric indices, and serum lipid indices in diabetic subjects by Pearson's two-tailed correlation analysis are summarized on Table 2. Those risk factors which had shown significant positive correlation with serum ferritin when diabetic subjects'data were analysed without regard to the nature of antidiabetic therapy, was repeated using medication as a key variable. There was a significant positive correlation $(\mathrm{p}<0.05)$ between serum ferritin and each of total cholesterol and TC/HDL in diabetic subjects undergoing drug therapy alone and between serum ferritin and each of plasma glucose, serum total cholesterol and serum triglyceride in subjects receiving diet therapy alone. There was no significant difference between serum ferritin and any risk factor in patients on combination therapy (oral drug + diet). The inference is that correlation between serum ferritin and other diabetes risk factors is affected by type of therapy.

Table 1: Serum Ferritin Levels According to Diabetes Medication

\begin{tabular}{llll}
\hline Group & Number & Treatment & $\begin{array}{c}\text { Serum Ferritin } \\
(\mathbf{n g} / \mathbf{m l})\end{array}$ \\
\hline A & 53 & Oral Drug & $80.72 \pm 7.12$ \\
B & 28 & Diet only & $78.52 \pm 9.68$ \\
C & 43 & Oral drug + Diet & $54.37 \pm 6.21^{\mathrm{ab}}$ \\
\hline
\end{tabular}

Tabulated values are means \pm SD; Statistical significance between means was assessed using one-way analysis of variance followed by Duncan's test as a post-analysis of variance test

${ }^{a}$ statistically significant difference at $\mathrm{p}<0.05$ compared to the group A

${ }^{b}$ statistically significant difference at $\mathrm{p}<0.05$ compared to the group B

Table 2: Pearson's Correlation between Serum Ferritin and Some Risk Factors in Diabetic subjects Receiving Different therapy

\begin{tabular}{lllllllll}
\hline Parameters & \multicolumn{2}{l}{ All cases } & \multicolumn{3}{l}{ Oral drug only } & \multicolumn{2}{c}{ Diet only } & \multicolumn{2}{c}{ Oral drug + Diet } \\
& $\mathrm{R}$ & $\mathrm{p}$-value & $\mathrm{R}$ & $\mathrm{p}$-value & $\mathrm{R}$ & $\mathrm{p}$-value & $\mathrm{r}$ & $\mathrm{p}$-value \\
\hline Age & -.003 & .977 & -.071 & .612 & -.042 & .838 & .072 & .657 \\
Glucose & $.248^{* *}$ & .005 & .131 & .351 & $.484 *$ & .012 & .023 & .888 \\
BMI & .067 & .460 & .120 & .396 & .129 & .529 & -.217 & .178 \\
Systolic BP & $.185^{*}$ & .040 & .003 & .981 & .228 & .273 & -.079 & .628 \\
Diastolic BP & $.215^{*}$ & .017 & .084 & .553 & .146 & .487 & .227 & .159 \\
TC & $.368^{*} *$ & .000 & $.349 *$ & .010 & $.433 *$ & .027 & .280 & .080 \\
TG & $.275^{*} *$ & .002 & .078 & .592 & $.421 *$ & .032 & .229 & .167 \\
HDL & .098 & .276 & -.013 & .924 & .165 & .419 & -.014 & .931 \\
TC/HDL & $0.338^{* *}$ & .000 & $.408 *$ & .002 & .161 & .432 & .256 & .110 \\
\hline
\end{tabular}

*Correlation is significant at the 0.05 level (2-tailed)

**Correlation is significant at the 0.01 level (2-tailed)

\section{DISCUSSION}

Epidemiological studies have reported positive associations of serum ferritin concentrations with cardiovascular risk factors, and risk of type 2 diabetes $(12,13)$. A significant positive and strong association between serum ferritin and fasting plasma glucose as obtained in this study is a strong indication to show that an elevated iron store is associated with diabetes risk. Serum ferritin is a major iron storage protein in the body and is by far the most commonly used indicator/index of body iron stores in epidemiological studies $(14,15)$. Our previous study provides evidence that the serum ferritin level is higher for type 2 diabetic patients and is correlated with some diabetes/cardiovascular risk factors including fasting blood glucose, systolic and diastolic blood pressure, triglyceride, total cholesterol and TC/HDL. This is in agreement with the report of several researchers in other countries of the world. For example, Sharifi et al. (16) found a significant correlation between serum ferritin and blood pressure, fasting plasma glucose, triglyceride and total cholesterol but not with BMI; Smotra and Kudyar, (6) reported significant correlation between serum ferritin and blood pressure, total 
cholesterol, triglyceride and uric acid levels; Wrede et al. (17) reported positive correlation between serum ferritin and BMI, TC, systolic and diastolic blood pressure.

The precise molecular mechanisms underlying the pathogenesis of iron-overload-related diabetes are poorly understood $(7,14)$. Although the exact mechanism of iron-induced diabetes is uncertain, it is likely to be mediated by three key mechanisms including insulin deficiency, insulin resistance, and hepatic dysfunction (18, 19). Excess iron impairs pancreatic $\beta$ cell function and causes $\beta$ cell apoptosis through increasing oxidative stress and thus results in impaired insulin synthesis and excretion $(20,14)$. The mechanisms for insulin resistance include the possibility of iron overload causing resistance directly or through hepatic dysfunction (21, 18). Dongiovianni et al. (8) investigated the effect of iron depletion by deferoxamine on insulin signaling and glucose uptake in HepG2 hepatocytes and in rat liver; and found that deferoxamine induced the constitutive glucose transporter Glut1 and the insulin receptor. Thus, iron depletion by deferoxamine up-regulates glucose uptake and increases insulin receptor activity and signaling in hepatocytes in vitro and in vivo. The generality of the epidemiological studies that reported strong association between elevated iron stores and increased risk for type 2 diabetes appear not to have taken the aetiological effect of the therapeutic regimen into critical consideration in the course of interpreting the observed relationship. In the present study, it was observed that oral drug or diet alone engendered higher ferritin than a combination and therefore less efficacious since high ferritin is a predictor of type 2 DM. Therefore, in terms of ferritin, a combination therapy is better. Also, correlation between body iron stores and diabetes risk factors is dependent on the type of medication for diabetes management, being pronounced with the use of combination therapy. Although the mechanism behind these findings was not elucidated in the present study, it could be attributed to the synergistic effect of drug and diet on the outcome of diabetes.

Metformin is considered a cornerstone in the treatment of diabetes and is the most frequently prescribed first line therapy for individuals with type 2 diabetes (22). A 2006 consensus statement from the American Diabetes Association (ADA) and the European Association for the Study of Diabetes (EASD), updated in 2009, proposed that metformin therapy (in the absence of contraindications) be initiated, concurrent with lifestyle intervention, at the time of diabetes diagnosis (23). Metformin appears to have effects in ameliorating oxidative stress and inflammation; rendering it an attractive tool for prevention of insulin resistance and diabetes (24). The role of diet (diet that is low in total fat, saturated fat, cholesterol, simple sugar, iron especially haem iron; but high in polysaturated fat, complex carbohydrate, and fibre) in the management of diabetes cannot be over emphasised. The disproportionate consumption of foods high in saturated fat, and high in simple sugars at the expense of foods high in complex carbohydrate and unsaturated fat has the potential of inducing abnormal metabolic processes in a normal healthy individual and to promote degenerative diseases such as type 2 DM, obesity, hypertension, or cardiovascular disease (25). High fat diet has been shown to be responsible for decreased insulin stimulated glucose transport and depressed intracellular glucose metabolism (26). Saturated fat has been shown to be responsible for elevation of hepatic iron retention which could be responsible for abnormalities of glucose metabolism $(27,25)$.

The general increases in consumption of calories, and specifically of refined carbohydrates and fructose, is clear and correlates positively with an alarming increase in metabolic syndrome (28). Fructose consumption is responsible for insulin resistance and abnormal glucose tolerance (29). In addition to contributing to metabolic abnormalities, the consumption of fructose has been reported to affect homeostasis of numerous trace elements, for e.g, fructose has been shown to increase iron absorption in humans and experimental animals (30). Abnormalities in glucose/insulin system when fructose is consumed could result from the coexistence of few conditions including elevated iron and generation of reactive oxygen species. Decrease in the consumption of total fat, saturated fat, cholesterol, salt, simple sugars, and an increase in the consumption of polyunsaturated fat, complex carbohydrate, and fibre has been reported to decrease risk factors associated with degenerative diseases (25). Reduction of liver iron decreases reactive oxygen species which in turn ameliorates the glucose/insulin system (25).

Epidemiological studies have established a link between increased dietary iron intake, particularly eating red meat, and increased body iron stores, and the development of diabetes $(14,19)$. For most people, iron is obtained mainly from dietary sources and then stored in the body (21). Dietary iron is present in either the haem or non-haem form (31). Prospective studies that evaluated the relationships between red meat (the major source of haem iron) and the risk of type 2 diabetes found positive association between haem iron and type 2 DM $(32,33)$. Because haem iron intake was found to be associated with higher body iron stores in previous studies $(34,35)$, high levels of body iron stores may mediate the association of increased risk of T2DM with chronically high haem iron intake (14). Reports from epidemiological studies have indicated that reduction of serum ferritin levels by bloodletting/ use of iron chelation therapy or reduction of red meat intake all have beneficial outcomes for delaying or preventing diabetic complications. 


\section{CONCLUSION}

Evidence has been presented in this study to show that Predictive effect of ferritin was strongly affected by type of diabetes medication. Combination therapy was more efficacious in reducing serum ferritin than either drug or diet alone suggesting that combination therapy may be more efficacious in the management of type $2 \mathrm{DM}$. There is therefore, the need to adhere strictly to diet regimen along side with taking oral medication as and when due, in order to achieve optimal goal in the management of type $2 \mathrm{DM}$.

Conflict of interest statement: The authors declare no conflict of interest.

\section{REFERENCES}

[1] R.K. Koshy, S. Raj, B. Kapoor, and M. Azamthulla, Anti hyperglycemic activity of Elytraria Acaulis Lind on StreptozotocinInduced diabetic rats, Medicinal and Aromatic Plants, 1, 2012, 103

[2] R. Wood, and E.C. O’Neill, Resistance training in type 2 diabetes: impact on areas of metabolic dysfunction in skeletal muscle and potential impact on bone, Journal of Nutrition and Metabolism 2012, 2012, 268197.

[3] S.A. Hussain, and B.H. Marouf, Flavonoids as alternatives in treatment of type 2 diabetes mellitus, Academia Journal of Medicinal Plants, 1(2), 2013, 031-036.

[4] S. Halimi, A. Schweizer, B. Minic, and S. Dejager, Combination treatment in the management of type 2 diabetes: focus on vildagliptin and metformin as a single tablet, Vascular Health Risk Management, 4(3), 2008, 481-492.

[5] S. Zanuso, A. Jimenez, G. Pugliese, G. Corigliano, and S. Balducci, "Exercise for the management of type 2 diabetes: a review of the evidence", Acta Diabetology, 47 (1), 2010, 15-22.

[6] S. Smotra, and R.P. Kudyar, Relationship between serum ferritin and type 2 diabetes mellitus, Science, 10(4), $2008,170-174$.

[7] J. S. Gabrielsen, Y. Gao, J. A. Simcox, J. Huang, D. Thorup, D. Jones, R. C. Cooksey, D. Gabrielsen, T. D. Adams, S. C. Hunt, P. N. Hopkins, W .T. Cefalu, and D. A. McClain, Adipocyte iron regulates adiponectin and insulin sensitivity, Journal of Clinical Investigation, 122(10), 2012, 3529-3540.

[8] P. Dongiovanni, L. Valenti, A.L. Fracanzani, S. Gatti, G. Cairo, and S. Fargion, Iron depletion by deferoxamine up-regulatrs glucose uptake and insulin signalling in hepatoma cells and in rat liver, American Journal of Pathology, 172, $2008,738-747$.

[9] P. Trinder, Enzymatic colorimetric methods, Annals of Clinical Biochemistry, 6, 1969, $24-27$.

[10] D. White, D. Kramer, G. Johnson, and H. Hamilton, Enzymatic immunoassay for the quantitative determination of ferritin concentration in serum, American Journal of Clinical Pathology, 72, 1986, 346.

[11] P.S. Bachorik, and J.J. Albers, Precipitation Methods for quantification of lipoprotein, Methods in Enzymology, 129, 1986, 78100 .

[12] N.G. Forouhi, A.H. Harding, M. Allison, M.S. Sandhu, A. Welch, R. Luben, S. Bingham, K.T. Khaw, and N.J. Wareham, Elevated serum ferritin levels predict new-onset type 2 diabetes: results from the EPIC-Norfolk prospective study, Diabetologia, 50(5), 2007, 949-956.

[13] F. Sharifi, A. Ziaee, A. Feizi, N. Mousavinasab, A. Anjomshoaa, and P. Mokhtari, Serum ferritin concentration in gestational diabetes mellitus and risk of subsequent development of early postpartum diabetes mellitus, Diabetes Metabolic Syndrome and Obesity, 3, 2010, 413-419.

[14] W. Bao, Y. Rong, S. Rong, and L. Liu, Dietary iron intake, body iron stores, and the risk of type 2 diabetes: a systematic review and meta-analysis, British Medial Counsel of Medicine, 10, 2012, 119.

[15] J. Montonen, H. Boeing, A. Steffen, R. Lehmann, A. Fritsche, H.G. Joost, M.B. Schulze, and T. Pischon, Body iron stores and risk of type 2 diabetes: results from the European Prospective Investigation into Cancer and Nutrition (EPIC)-Potsdam study, Diabetologia, 55(10), 2012, 2613-2621.

[16] F. Sharifi, N.M. Nasab, and H.J. Zadeh, Elevated serum ferritin concentrations in prediabetic subjects, Diabetes and Vascular Disease Research, 5 (1), 2008, 15-18.

[17] C.E. Wrede, R. Buettner, L.C. Bollheimer, J. Scholmerich, K.D. Palitzsch, and C. Hellerbrand, Association between serum ferritin and the insulin resistance syndrome in a representative population, Europian Journal of Endocrinology, 154, 2006, 333340.

[18] S. Swaminathan, V.A. Fonseca, M.G. Alam, and S.V. Shah, The role of iron in diabetes and its complications, Diabetes Care, 30(7), 2007, 1926-1933.

[19] Z. Zhao, S. Li, G. Liu, F. Yan, X. Ma, Z. Huang, and H. Tian, Body iron stores and heme-iron intake in relation to risk of type 2 diabetes: a systematic review and meta-analysis, PLOS ONE 7(7), 2012, e41641. doi:10.1371/journal.pone.0041641.

[20] R.C. Cooksey, D. Jones, and S. Gabrielsen, Dietary iron restriction or iron chelation protects from diabetes and loss of beta-cell function in the obese (ob/ob lep-/-) mouse, American Journal of Physiology and Endocrinology Metabolism, 298(6), 2010, 1236-1243.

[21] S.N. Rajpathak, J.P. Crandall, J. Wylie-Rosett, G.C. Kabat, T.E. Rohan, and F.B. Hu, The role of iron in type 2 diabetes in humans, Biochimica et Biophysica Acta ,1790(7), 2009, 671-681.

[22] J. De Jager, A. Kooy, P. Lehert, M.G. Wulffelé, J.V. Kolk, D. Bets, J. Verburg, A.J.M. Donker, and C.D.A. Stehouwer, Long term treatment with metformin in patients with type 2 diabetes and risk of vitamin b-12 deficiency: randomised placebo controlled trial, British Medical Journal, 340, 2010, 2181.

[23] D.M. Nathan, J.B. Buse, M.B. Davidson, E. Ferrannini, R.R. Holman, and R. Sherwin, Medical management of hyperglycaemia in type 2 diabetes: a consensus algorithm for the initiation and adjustment of therapy: a consensus statement of the American Diabetes Association and the European Association for the Study of Diabetes, Diabetes Care, 32(1), 2009, $193-203$.

[24] M.Z. Gad, N.A. Ehssan, M.H. Ghiet, and L.F. Wahmanb, Pioglitazone versus metformin in two rat models of glucose intolerance and diabetes, Pakistan Journal of Pharmaceutical Science, 23(3), 2010, 305-312.

[25] M. Fields, Nutritional Factors Adversely Influencing the Glucose/Insulin System, Journal of the American College of Nutrition, 17(14), 1998, 317-321

[26] C.H. Tsunehara, D.L. Leonetti, and W.Y. Fujimoto, Animal fat and cholesterol intake is high in men with IGT progressing to Non-insulin- dependent diabetes mellitus (NIDDM), Diabetes, 40, 1991, 427A.

[27] P.E. Johnson, H.C. Lukaski, and E.D. Kouynta, Effects of stearic acid and beef tallow in iron utilization by the rat, Proceedings of the Society for Experimental Biology and Medicine, 200, 1992, 480-486. 
[28] H. Basciano, L. Federico, and K. Adeli, Fructose, insulin resistance, and metabolic dyslipidemia, Nutrition and Metabolism, 2, 2005, 1-37.

[29] J.M. Olefsky, and A. Nolan, Insulin resistance and non-insulin dependent diabetes mellitus: Cellular and molecular mechanism, American Journal of Clinical Nutrition, 61(l), 1995, 980S-986S.

[30] J.T. Holbrook, J.C. Smith, and S. Reiser, Dietary fructose or starch: effects on copper, zinc, iron, manganese, calcium and magnesium balances in humans, American Journal of Clinical Nutrition, 49, 1989, 1290-1294.

[31] I. De Domenico, D. McVey Ward, and J. Kaplan, Regulation of iron acquisition and storage: consequences for iron-linked disorders, National Review of Molecular Cell Biology, 9(1), 2008, 72-81.

[32] D. Aune, G. Ursin, and M.B. Veierod, Meat consumption and the risk of type 2 diabetes: a systematic review and meta-analysis of cohort studies, Diabetologia, 52(11), 2009, 2277-2287.

[33] A. Pan, Q. Sun, A.M. Bernstein, M.B. Schulze, J.E. Manson, W.C. Willett, and F.B. Hu, Red meat consumption and risk of type 2 diabetes: 3 cohorts of US adults and an updated meta-analysis, American Journal of Clinical Nutrition, 94(4), 2011, 10881096.

[34] D.J. Fleming, P.F. Jacques, G.E. Dallal, K.L. Tucker, P.W. Wilson, and R.J. Wood, Dietary determinants of iron stores in a freeliving elderly population: The Framingham Heart Study, American Journal of Clinical Nutrition, 67(4), 1998, 722-733.

[35] J.M. Liu, S.E. Hankinson, M.J. Stampfer, N. Rifai, W.C. Willett, and J. Ma, Body iron stores and their determinants in healthy postmenopausal US women, American Journal of Clinical Nutrition, 78(6), 2003, 1160-1167. 